\title{
Interstitielle Lungenerkrankungen - historische Entwicklung, Status quo und Ausblick
}

\author{
Interstitial Lung Diseases - Historical Development, Current Status, \\ Future Prospects
}

Autoren

Institute
J. Behr ${ }^{1}$, U. Costabel ${ }^{2}$

Medizinische Klinik und Poliklinik I, Schwerpunkt Pneumologie, Klinikum der Universität München

Abt. Pneumologie/Allergologie, Ruhrlandklinik Essen eingereicht 25.7.2010

akzeptiert 30.7.2010

\section{Bibliografie}

DOI http://dx.doi.org/ 10.1055/s-0030-1255627 Pneumologie 2010; 64: 573-576 @ Georg Thieme Verlag KG Stuttgart · New York ISSN 0934-8387

\section{Korrespondenzadresse}

Prof. Dr. med. Jürgen Behr Medizinische Klinik und Poliklinik ।

Schwerpunkt Pneumologie Klinikum der Universität München

Marchioninistr.15 81377 München juergen.behr@med.unimuenchen.de

\section{Zusammenfassung \\ $\nabla$}

Die Erstbeschreibung der Sarkoidose wie auch der fibrosierenden Lungenerkrankungen geht zurück auf histopathologische Beobachtungen im 19. Jahrhundert. Seither werden zahlreiche unterschiedlicher Krankheitsentitäten identifiziert, die unter dem Oberbegriff der diffusen Lungenparenchymerkrankungen zusammengefasst werden. Gemeinsam ist diesen die Manifestation im Interstitium der Lunge, wobei ein breites Spektrum von überwiegend entzündlichen bis hin zu rein fibrosierenden Prozessen beobachtet wird. Erstere sprechen naturgemäß auf anti-entzündliche Therapie in der Regel gut an, während für letztere effektive Medikamente fehlen. Gentechnologische Untersuchungen haben das pathophysiologische Verständnis der fibrosierenden Lungenerkrankungen grundlegend erneuert. Im Bereich der Früherkennung und Differenzialdiagnose stellt die hochauflösende Computertomografie einen entscheidenden Fortschritt dar. Hinsichtlich der Therapie der Lungenfibrose ergeben sich erste positive Befunde für hoch dosierte N-Acetylcystein sowie für Pirfenidon. Das verbesserte Verständnis der Pathophysiologie lässt hoffen, dass zukünftig zielgerichtete Therapieansätze erfolgreich getestet werden können.

\section{Einleitung \\ $\nabla$}

Die Erstbeschreibung fibrosierender interstitieller Lungenerkrankungen geht zurück in das ausgehende 19. Jahrhundert, als G. E. Rindfleisch (1897) und P. von Hansemann (1898) Erkrankungen mit der Bezeichnung „Cirrhosis cystica pulmonum“ und „Lymphangitis reticularis“ beschrieben. Mehr als 30 Jahre später berichteten die Pathologen Hamman und Rich erstmals über Fälle einer fulminant verlaufenden, diffusen, interstitiellen Lungenfibrose, wofür später die Bezeichnung „Hamman-Rich-Syndrom“ geprägt wurde. Die erste mo-

\section{Abstract \\ $\nabla$}

Sarcoidosis and lung fibrosis were first described by histopathology in the $19^{\text {th }}$ century. Since then a large number of different forms of diffuse parenchymal lung diseases has been identified. Although all these diseases manifest in the lung interstitium, there is a wide range of predominanthy inflammatory to purely fibrotic disease processes. Accordingly, anti-inflammatory treatment is successful in the former, whereas for the latter an effective medical therapy is lacking. Gene technology has recently led to results which have fundamentally changed our understanding of the pathophysiology of fibrosing lung disease. Early and differential diagnosis has much improved with the help of high-resolution computed tomography. Recent clinical trials in patients with idiopathic pulmonary fibrosis have shown at least some effectiveness for the antioxidant treatment approach using high-dosed $\mathrm{N}$-acetylcysteine as well as for the use of pirfenidone. Importantly, these studies have proven that well-designed treatment trials are feasible in this patient population. This has kindled the hope that, based on a better understanding of the pathophysiology, new targeted therapies will prove to be successful in the future.

derne Klassifikation der interstitiellen Lungenerkrankungen wurde von Liebow, Carrington und Gaensler in den 60er- und 70er-Jahren des 20. Jahrhunderts erarbeitet. Bereits damals hatte man grundlegende Tatsachen erkannt, die bis heute ihre Richtigkeit behalten haben:

1. Das histologische Befundmuster ist von prognostischer Bedeutung, wobei insbesondere das Ausmaß der Fibrosierung mit einem ungünstigen Verlauf assoziiert ist.

2. Einige Patienten mit interstitiellen Lungenerkrankungen reagieren auf Kortikosteroidthera- 


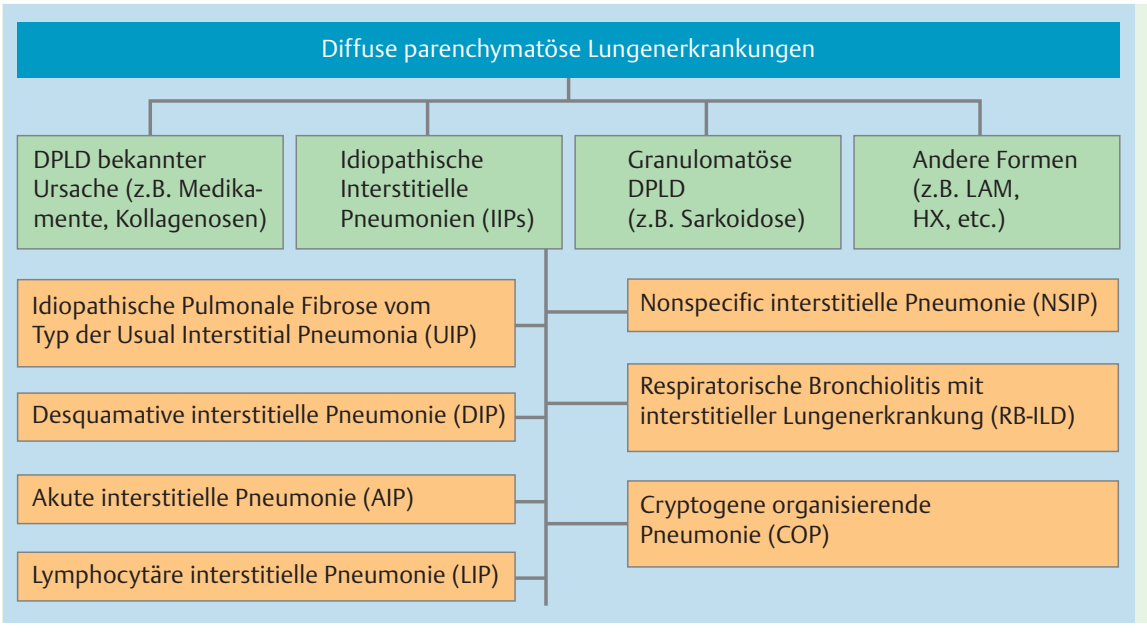

Abb. 1 Internationale Konsensusklassifikation der diffusen Lungenparemchymerkrankungen. pie und das Absetzen der Kortikosteroide kann eine Exazerbation der Erkrankung auslösen.

3. Patienten, die das histologische Befundmuster einer „usual interstitial pneumonia“ (UIP) aufweisen, erleiden einen in der Regel progressiven, tödlichen Krankheitsverlauf und sprechen selten oder nie auf Kortikosteroide an.

Seit diesen Erstbeschreibungen wurde eine weite Differenzialdiagnose möglicher Ursachen und Assoziationen von interstitiellen Lungenerkrankungen beschrieben, sodass heute weit über 150 unterschiedliche Krankheitsbilder differenziert werden, die mit einer interstitiellen Lungenerkrankung einhergehen können. Ein umfassendes Klassifikationsschema wurde von den internationalen Fachgesellschaften ATS/ERS im Jahr 2002 publiziert (๑ Abb. 1).

Dabei werden Erkrankungen bekannter Ursache (z. B. medikamentös-toxische interstitielle Lungenerkrankungen), granulomatöse Erkrankungen (z.B. Sarkoidose) und spezielle Entitäten (z.B. Langerhanszell-Histiozytose oder Lymphangioleiomyomatose) sowie die große Gruppe der idiopathischen interstitiellen Pneumonien unterschieden. Letztere umfasst sieben Subspezialitäten, wobei die idiopathische Lungenfibrose mit dem histologischen Befundmuster der usual interstitial pneumonia die häufigste Form darstellt und gleichzeitig den ungünstigsten Krankheitsverlauf aufweist. Aufgrund der breiten Differenzialdiagnose und der häufigen Assoziation mit Systemerkrankungen und Stoffwechselstörungen sind die interstitiellen Lungenerkrankungen ein wichtiger Bestandteil der allgemeinen inneren Medizin. Trotz der ausgesprochenen Heterogenität der Krankheitsgruppe der interstitiellen Lungenerkrankungen lassen sich auch gemeinsame Charakteristika feststellen, welche einerseits in den veränderten physiologischen Verhältnissen mit restriktiver Ventilationsstörung und eingeschränktem Gasaustausch liegen, andererseits in der gemeinsamen Endstrecke des irreversiblen, fibrotischen Lungenparenchymumbaus zu sehen sind. Allerdings variieren die verschiedenen Krankheitsaspekte Entzündung, Granulombildung, Fibroproliferation und fibrotische Narbenbildung zwischen den verschiedenen Krankheitsentitäten erheblich, woraus auch eine unterschiedliche therapeutische Ansprechbarkeit resultiert.

Als Erstbeschreiber der Sarkoidose, neben der idiopathischen Lungenfibrose die häufigste interstitielle Lungenerkrankung, gilt der englische Arzt J. Hutchinson, der 1869 erstmals Hautveränderungen beschrieb. Der Name Sarkoidose stammt von C. Boeck (1897), als er die Hautveränderungen mit dem Begriff „multiples benignes Sarkoid“ bezeichnete. Im frühen 20. Jahrhundert finden sich Erstbeschreibungen weiterer Organmanifestationen (Knochenverän- derungen durch Kienbock/Kreibig/Jüngling, Uveitis durch Schuhmacher/Heerfordt/Behring). Mit zunehmender Anwendung von Röntgenuntersuchungen wurde der systemische Charakter der Erkrankung erkannt. In der zweiten Hälfte des 20. Jahrhunderts wurden die Röntgenstadieneinteilung und Aktivitätsmarker entwickelt, wobei hier der deutsche Sarkoidosepionier K. Wurm aus Höchenschwand hervorzuheben ist. Immunologische Befunde wie die Anergie gegen Tuberkulin, die lokalen Aktivitätsphänomene wie Vermehrung aktivierter T-Lymphozyten vom Th1-Typ in den Lungen mit entsprechender Cytokinbildung und der Nachweis aktivierter Makrophagen, welche TNF-alpha und andere für die Granulombildung bedeutsame Zytokine bilden, wurden erst in den letzten 30 Jahren beschrieben. Diese neuen Erkenntnisse waren insbesondere auch der Einführung der BAL in Forschung und Klinik zu verdanken.

\section{Pathophysiologie}

$\nabla$

Angesichts der beschriebenen Heterogenität der Krankheitsgruppe verwundert es nicht, dass auch die pathophysiologischen Grundlagen sehr unterschiedlich sind. So gibt es Erkrankungen mit überwiegend entzündlichem Hintergrund, wie z.B. die exogen-allergische Alveolitis oder auch Beteiligungen des Lungenparenchyms im Rahmen von Kollagenosen. Andererseits sind Zigarettenrauch-assoziierte Erkrankungen wie die respiratorische Bronchiolitis mit interstitieller Lungenerkrankung (RB-ILD) und die desquamative interstitielle Pneumonie abzugrenzen. Zahlreiche medikamentös-toxische Reaktionen (z.B. Bleomycinlunge, Amiodaronlunge) sind ebenfalls durch ausgeprägte inflammatorische Krankheitsmechanismen geprägt.

Wichtige Fortschritte wurden auch im Verständnis der idiopathischen pulmonalen Fibrose (IPF) in den letzten Jahren erzielt. So konnten Surfactant-Protein-C- und Surfactant-Protein-A-Mutationen als Ursache der familiären Form der idiopathischen Lungenfibrose identifiziert werden. Sowohl familiäre als auch sporadische Fälle von idiopathischer Lungenfibrose können durch Mutationen im Telomerasegen ausgelöst werden. Im Falle des Hermanski-Pudlak-Syndroms liegt offenbar ein Gendefekt im Bereich des lysosomalen Metabolismus der Alveolartyp-II-Zellen vor. All diesen genetischen Anomalien ist gemeinsam, dass sie über einen gestörten Metabolismus der Typ-II-Pneumozyten zu einer vorzeitigen Apoptose dieser Zellen führen, die ihrerseits eine Hyperplasie der verbleibenden Pneumozyten auslöst. Hierdurch wird zum einen die suprimierende Kontrolle der Epithelzellen über die interstitiellen 


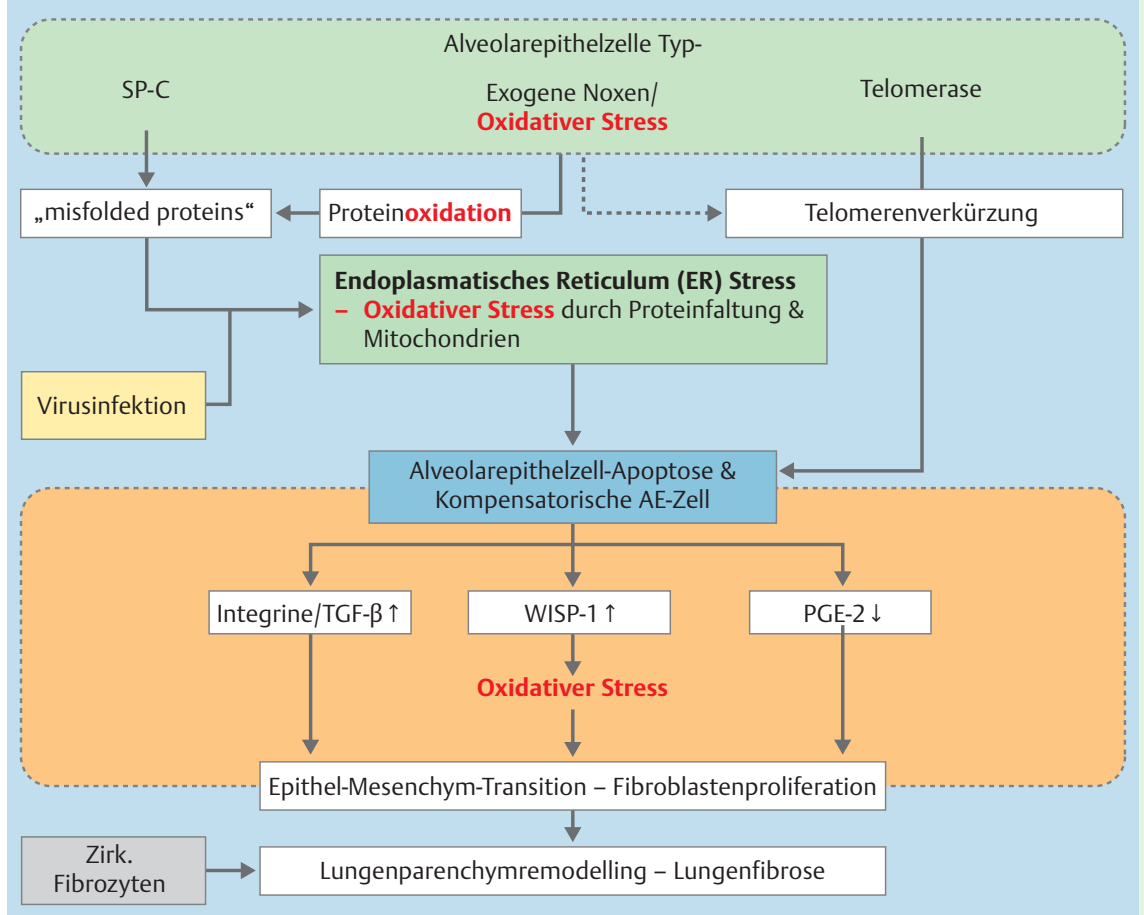

Abb. 2 Moderne Pathophysiologie fibrosierender Lungenerkrankungen.

Fibroblasten (via PGE2) nicht mehr gewährleistet und es resultiert ein Fibroblastenwachstum vor Ort; zum anderen werden gleichzeitig embryonale Reparaturmechanismen reaktiviert, die zu einer fehlgesteuerten, fibroproliferativ geprägten „Wundheilung“ im Alveolarbereich führen ( $\bullet$ Abb. 2).

Ausgehend von diesen neuen Erkenntnissen ist klar, dass die Entzündungsreaktion bei diesen Krankheitsbildern nur eine untergeordnete Rolle spielt, während der oxidative Stress auf allen Ebenen der Krankheitsentwicklung eine zentrale Rolle zu spielen scheint. Neben der direkten Proliferation ortständiger Fibroblasten scheinen zwei weitere Mechanismen zum fibrotischen Lungenparenchymumbau beizutragen, einerseits die Epithel-Mesenchym-Transition, bei der sich Epithelzellen unter den Einfluss bestimmter Zytokine (z.B. TGF- $\beta$ ) in Mesenchymzellen umwandeln; andererseits kommt es zur Einwanderung von mesenchymalen Progenitorzellen aus dem Knochenmark bzw. von zirkulierenden Fibrozyten aus dem Blut in das Lungenparenchym, wo sie den fibroproliferativen Prozeß modifizieren. Noch lässt sich der Beitrag der verschiedenen Fibrogenesemechanismen zur Entstehung der Lungenfibrose nicht quantifizieren, das Hauptgewicht scheint jedoch auf der Proliferation ortständiger Lungenfibroblasten, bedingt durch eine unzureichende supramierende Kontrolle durch das geschädigte Alveolarepithel, zu liegen.

Basierend auf diesen jüngsten Fortschritten im pathophysiologischen Verständnis der Lungenfibrose ergeben sich neue therapeutische Ansatzpunkte, die aktuell in klinischen Studien erprobt werden.

\section{Diagnostik}

\section{$\nabla$}

In Anbetracht der breiten Differenzialdiagnose der interstitiellen Lungenerkrankungen ist für eine korrekte Diagnosestellung eine umfassende internistische Abklärung jedes einzelnen Patienten erforderlich, die die verschiedenen Aspekte einschließlich häuslicher, privater und beruflicher Schadstoffexposition, Medikamentenanamnese, Familienanamnese und gezielten Suchens nach übergeordneten Systemerkrankungen umfasst. Eine zentrale und in den letzten Jahren zunehmende Rolle der Diagnosestellung spielt die hochauflösende Computertomografie der Lunge. Der technische Fortschritt in diesem Bereich hat zu einer enormen Verbesserung der Bildqualität geführt, sodass nun Befundmuster im hochauflösenden Computertomogramm abgrenzbar sind, die entsprechenden Krankheitsbildern zugeordnet werden können. So ist der Nachweis von basal betontem, subpleuralem Honigwabenmuster mit retikulärer interstitieller Zeichnungsvermehrung und Traktionsbronchiektasen bei gleichzeitigem Fehlen von Milchglastrübungen als charakteristisch für eine usual interstitial pneumonia anzusehen. Dieses Muster allerdings kann entweder als idiopathische, also eigenständige Erkrankung, oder im Zusammenhang mit einer exogen-allergischen Alveolitis oder einer Kollagenose auftreten. Dementsprechend lässt sich aus dem Befundmuster im hochauflösenden Computertomogramm noch keine definitive Diagnose ableiten, vielmehr lässt sich nur in Zusammenschau aller klinischen und apparativen Untersuchungsergebnissen die korrekte Diagnose stellen. Ähnlich verhält es sich mit der sogenannten nicht-spezifischen interstitiellen Pneumonie (NSIP), die häufig z.B. bei Sklerodermiepatienten beobachtet wird, die aber auch bei der exogen-allergischen Alveolitis vorkommt oder als idiopathische, also eigenständige Erkrankung auftritt. Dementsprechend ist neben der Erkennung des pulmonalen Fibrosemusters vor allem der Ausschluss oder Nachweis eines exogenen Auslösers oder einer übergeordneten Systemerkrankung von entscheidender Bedeutung für die richtige Diagnosestellung bei diesen Krankheitsbildern. Zu diesem Zweck können neben immunologischen Untersuchungen im Blut auch die Bronchoskopie mit bronchoalveolärer Lavage und transbronchialer Lungenbiopsie wichtige Hinweise auf das zugrunde liegende Krankheitsbild liefern oder dazu beitragen, mögliche Differenzialdiagnosen auszuschließen.

Die chirurgische Biopsie als Gold-Standard der Diagnostik interstitieller Lungenerkrankungen wurde in Anbetracht der Komplexizität der verschiedenen Krankheitsbilder in Frage gestellt. Auch die chirurgische Lungenbiopsie kann letztlich nur ein histologisches 
Befundmuster liefern, nicht jedoch eine definitive Diagnose. Letztere ergibt sich aus der Gesamtschau der klinischen, funktionsanalytischen, bronchoskopischen und ggf. histologischen Befunde. In Anbetracht der verbesserten Bildgebungsqualität der hochauflösenden Computertomografie ist bei der Diagnosestellung einer idiopathischen Lungenfibrose die chirurgische Lungenbiopsie nur noch in etwa 20 -30\% der Fälle erforderlich. Dies vor allem bei den Patienten, bei denen die hochauflösende Computertomografie keine eindeutige Zuordnung des Befundmusters erlaubt, meist im frühen Krankheitsstadium. Der neue Gold-Standard in der Diagnose der interstitiellen Lungenerkrankungen ist dementsprechend die multidisziplinäre Fallkonferenz, in deren Rahmen der Pneumologe, der Pathologe und der Radiologe die erhobenen Befunde diskutieren und letztendlich einem spezifischen Krankheitsbild zuordnen.

\section{Therapie}

Trotz der bemerkenswerten Fortschritte in unserem pathophysiologischen Verständnis der interstitiellen Lungenerkrankungen sowie in der Diagnostik und Klassifikation sind die Fortschritte hinsichtlich der Therapie noch relativ gering. Eine primär antientzündliche Behandlung mit Kortikosteroiden und zytotoxischen Substanzen vom Typ des Azathioprins oder Cyclophosphamids kann vor allem bei Erkrankungen mit im Vordergrund stehender inflammatorischer Komponente erfolgreich sein. Hierzu zählen die zelluläre Form der NSIP oder die kryptogen organisierende Pneumonie und auch die chronische eosinophile Pneumonie. Für die Behandlung der idiopathischen Lungenfibrose dagegen ist eine rein antiinflammatorische Therapie in der Regel nicht zu empfehlen. Neuere Untersuchungen zeigen allerdings, dass ein antioxidativer Therapieansatz mit hochdosiertem N-Acetylcystein den Abfall der Lungenfunktionsparameter Vitalkapazität und Diffusionskapazität im Verlauf eines Jahres signifikant stabilisieren und verzögern kann; insbesondere dann, wenn der fibrotische Lungenparenchymumbau noch nicht weit fortgeschritten ist. Zu diesem Ergebnis kam die prospektive, randomisierte, plazebokontrollierte multizentrische IFIGENIA-Studie, bei der $3 \times 600$ mg N-Acetylcystein im Vergleich zu Plazebo zusätzlich zu einer Basistherapie mit Prednison und Azathioprin bei 175 Patienten im Verlauf eines Jahres untersucht wurde. Keine eindeutige Antwort lieferte diese Studie auf die Frage, ob N-Acetylcystein alleine oder nur in Kombination mit Prednison und Azathioprin wirkt. Die Dreifachtherapie aus Prednison, Azathioprin und NAC entspricht der vollständigen Medikation während der Studie, während N-Acetylcystein der tatsächlich versus Plazebo getesteten Studienmedikation entspricht. Da eine Interaktion zwischen Prednison-Azathioprin auf der einen Seite und N-Acetylcystein auf der anderen Seite weder nachgewiesen ist noch sicher ausgeschlossen werden kann, ist die Evidenzlage für beide Regime als ähnlich zu betrachten. Als zweite Substanz, die eine gewisse Wirksamkeit bei der idiopathischen Lungenfibrose gezeigt hat, ist Pirfenidon zu nennen. Pirfenidon ist ein Pyrdonderivat mit antifibrotischer Wirkkomponente, insbesondere durch Hemmung von TGF$\beta$-Effekten. In mehreren klinischen Studien hat Pirfenidon einerseits den Abfall der Vitalkapazität im Verlaufe eines Jahres verlangsamt, andererseits das progressionsfreie Überleben verlängert. Allerdings konnte in einer Studie kein signifikanter Therapieeffekt nachgewiesen werden, sodass insgesamt widersprüchliche Studienergebnisse vorliegen. Betrachtet man alle Studien zusammen, so kann für Pirfenidon ein gewisser positiver Effekt auf das Fortschreiten als wahrscheinlich gelten, allerdings um den Preis von
Nebenwirkungen, die von gastrointestinalen Beschwerden über allgemeine Mattigkeit bis hin zu Photosensibilisierung reichen. Stellt man die beiden therapeutischen Optionen mit N-Acetylcystein und Pirfenidon einander gegenüber, so ergibt sich für N-Acetylcystein aufgrund der Tatsache, dass nur eine randomisierte, plazebokontrollierte Studie existiert, eine schwächere Evidenzlage im Vergleich zu Pirfenidon, bei dem wiederum das ungünstigere Nebenwirkungsprofil berücksichtigt werden muss. Darüber hinaus ist Pirfenidon vor der beantragten Zulassung noch nicht für Patienten außerhalb von Studien verfügbar. Daher müssen alle therapeutischen Optionen einschließlich der Teilnahme an einer Therapiestudie mit dem Patienten eingehend besprochen und diskutiert werden, um dann zu einer mit dem Patienten abgestimmten Entscheidung über das therapeutische Vorgehen zu gelangen. Hierbei ist insbesondere auch die Option der Lungentransplantation für geeignete Patienten von großer Bedeutung, da nur sie eine Verbesserung der respiratorischen Funktion und bei richtiger Patientenauswahl eine Verlängerung des Überlebens gewährleisten kann. Die Entwicklung der Lungentransplantation seit Einführung in den 80er-Jahren des 20. Jahrhunderts erlaubt heute auch Patienten mit Lungenfibrose 5-Jahres-Überlebensraten von ca. $60 \%$, was einer erheblichen Verbesserung der Prognose bei fortgeschrittener idiopathischer Lungenfibrose entspricht.

\section{Ausblick \\ $\nabla$}

Durch die einheitliche Klassifikation und Diagnosestellung der interstitiellen Lungenerkrankungen wurden in den letzten Jahren zunehmend internationale randomisierte, plazebokontrollierte Studien möglich, die einen wesentlichen Fortschritt auf diesem Gebiet darstellen und mit N-Acetylcystein und Pirfenidon als Therapieoptionen erste, wenn auch noch nicht befriedigende Ergebnisse geliefert haben. Zahlreiche antifibrotische Therapieansätze konnten allerdings bisher keine Erfolge vorweisen. So ergaben randomisierte, plazebokontrollierte Studien mit Interferon- $\gamma-1 b$, dem TNF-alpha-Antagonisten Etanercept, dem Tyrosinkinaseinhibitor Imatinib, dem Endothelinrezeptorantagonisten Bosentan letztlich jeweils negative Ergebnisse, sodass auch das antifibrotische Therapiekonzept offenbar nicht die Lösung für das Problem der Lungenfibrose darstellt. Als Zukunftsperspektive ist dementsprechend ein eher multimodales Therapiekonzept zu sehen, welches neben antientzündlichen auch antioxidative und antiproliferative Komponenten beinhalten müsste. Ähnlich wie bei onkologischen, neoplastischen Erkrankungen könnte eine solche Kombinationstherapie den ersehnten Erfolg für Patienten mit fibrosierenden interstitiellen Lungenerkrankungen ermöglichen. Eine weitere Alternative könnten zelluläre Therapiekonzepte darstellen, beispielsweise durch Zuführung entsprechend vorprogrammierter mesenchymaler Progenitorzellen. Auf diesem Gebiet sind jedoch noch viele Fragen unbeantwortet, die Zukunft hat aber bereits begonnen.

\section{Interessenkonflikte \\ $\nabla$}

J. Behr erhielt Honorare für Vorträge und Beratertätigkeit von Actelion, Bayer, Boehringer, Gilead, GlaxoSmithKline, Lilly und Pfizer. U. Costabel war als Principal Investigator und Mitglied des Steering Committee an Therapiestudien zur Prüfung von N-Acetylcystein (Firma Zambon) bzw. Pirfenidon (Firma Intermune) bei idiopathischer Lungenfibrose beteiligt. 